Proceedings of XIX International Scientific Conference "New Technologies and Achievements in Metallurgy, Material Engineering, Production Engineering and Physics", Częstochowa, Poland, June 7-8, 2018

\title{
Domain Structure and Magnetization Reversal Processes in Magnetic Alloys with Shape and Magnetocrystalline Anisotropy
}

\author{
I. Wnuk*, A. Przybye, K. Kutynia And J.J. WysŁocki \\ Częstochowa University of Technology, Institute of Physics, al. Armii Krajowej 19, 42-200 Częstochowa, Poland
}

\begin{abstract}
The mechanism of magnetization reversal processes in hard magnetic alloys with different type of magnetic anisotropy, i.e. shape (Alnico, $\mathrm{Fe}-\mathrm{Cr}-\mathrm{Co}$ ) and magnetocrystalline ( $\mathrm{Nd}-\mathrm{Fe}-\mathrm{B})$, were investigated on the basis of the rotational hysteresis energy and its integral calculated from the magnetic torque curves. Moreover, the measured angular dependence of the coercive field was analyzed within the framework of theoretical results for pinning and nucleation models. Additionally, observation of the magnetic domain structure was made by means of the powder pattern technique. All experimental data compared with appropriate theoretical models clearly indicate that the magnetic hardening mechanism in the studied magnets with (i) shape anisotropy (Alnico, $\mathrm{Fe}-\mathrm{Cr}-\mathrm{Co}$ ) occurs principally by the curling mechanism and (ii) magnetocrystalline anisotropy $(\mathrm{Nd}-\mathrm{Fe}-\mathrm{B})$ is based on the nucleation process.
\end{abstract}

DOI: 10.12693/APhysPolA.135.292

PACS/topics: hard magnetic materials, magnetic anisotropy, domain structure, magnetization reversal process

\section{Introduction}

Owing to the understanding of the mechanisms of the magnetization reversal processes, a further progress in the improvement of the magnetic properties of magnetically hard materials is possible. However, these processes are complex, and differences still exist in the theoretical description of the sources of the magnetic hardness of materials [1], whose measure is the coercivity. The occurrence of coercivity is associated with the existence of magnetic anisotropy. This anisotropy may be the anisotropy of shape, related to the geometrical shape of the ferromagnetic phase (as is the case of Alnico magnets [2, 3], or $\mathrm{Fe}-\mathrm{Cr}-\mathrm{Co}$ magnets [4]), or crystalline anisotropy, associated with the type of the crystal lattice of material (as in the case of ferrite [5], $\mathrm{Nd}-\mathrm{Fe}-\mathrm{B}$ [6] or $\mathrm{Sm}-\mathrm{Fe}-\mathrm{N}$ [7] magnets). The coercivity of presently manufactured magnets attains only approximately $10-20 \%$ of the theoretically determined value [8], which indicates a huge potential lying in these materials, that has not been used yet. It only remains for us to answer the question of how to do this. So far, the majority of studies has been focused chiefly on the examination of the effect of various factors (such as the manufacturing technology, chemical composition or phase structure) on the final magnetic properties of the magnets [1]. The mechanisms of magnetization reversal of these materials, that is the mechanisms which assure the obtaining of the optimum magnetic properties, are not fully understood. An important issue is also to obtain the information on whether or not

*corresponding author; e-mail: wnukiza@gmail.com the magnetization process is influenced by the type of magnetic anisotropy occurring in a particular magnetic material.

Therefore, the main objective of the studies presented in this article is chosen to be the determination and comparison of magnetization reversal mechanisms occurring in materials that are characterized by different shape and crystalline anisotropy. For comparison, the $\mathrm{Nd}-\mathrm{Fe}-\mathrm{B}$ alloy is selected as an example of material with crystalline anisotropy, and the Alnico and $\mathrm{Fe}-\mathrm{Cr}-\mathrm{Co}$ alloys are examples of shape anisotropy materials.

The additional objective of the study is to establish the domain structure and to determine the angular relationship of the coercivity of the magnetic materials examined.

\section{Material and experimental methods}

Some interesting information about the process of magnetization reversal of contemporary magnetically hard materials is provided by studies on the rotational hysteresis energy, $W_{r}$, as determined from the magnetic torque curves, $T$, in the rotational magnetic field.

The rotational hysteresis energy, $W_{r}$, is the measure of energy needed for a slow rotation of a ferromagnetic body by an angle of $360^{\circ}$ in a stationary magnetic field [8]. This energy is defined as the algebraic sum of areas formed by the positive and negative sections of the magnetic torque curve with the clockwise $(\mathrm{CW})$ rotation of the external magnetic field [8]:

$$
W_{r}=\int_{0}^{2 \pi} T(\Theta) \mathrm{d} \Theta,
$$

where $\Theta$ is the angle between the easy magnetization direction and the direction of the external magnetic field 
applied. If the magnetic field is rotated by $360^{\circ}$ in the anticlockwise $(\mathrm{ACW})$ direction, the two torque curves (for the $\mathrm{CW}$ and $\mathrm{ACW}$ rotations) contain between them an area which is numerically equal to the doubled value of the rotational energy [9]:

$$
W_{r}=\frac{1}{2}\left[\int_{0}^{2 \pi} T_{C W}(\Theta) \mathrm{d} \Theta-\int_{0}^{2 \pi} T_{A C W}(\Theta) \mathrm{d} \Theta\right],
$$

where $T_{C W}(\Theta)$ and $T_{A C W}(\Theta)$ denote the magnetic torque, as measured for external magnetic field rotation, clockwise and anticlockwise respectively.

Jacobs and Luborsky [10] also reported that interesting information about the magnetization reversal processes can be derived from the so-called integral of the rotational hysteresis, $R$ :

$$
R=\int_{0}^{2 \pi} \frac{W_{r}(H)}{M_{s}} \mathrm{~d}\left(\frac{1}{H}\right) .
$$

This integral, as defined by the formula below is a dimensionless quantity, being dependent only on the mechanism of magnetization of a given material.

The measurements of the torque curve, $T$, depending on the angle $\theta$ made by the direction of the external field an the axis of easy magnetization in the specimen, were performed at room temperature using a doublesuspension torsional anisometer in a magnetic field of up to $1985 \mathrm{kA} / \mathrm{m}$.

The domain structure was observed by using the powder pattern method. Disclosing the domain structure consisted in the application of a colloidal suspension of ferromagnetic $\mathrm{Fe}_{3} \mathrm{O}_{4}$ powder (ferrofluid) with a particle diameter below $1 \mu \mathrm{m}$ to the polished surface of specimens. So obtained domain images were photographed using a metallographic microscope.

The examination was carried out on the following magnetically hard alloys:

- $\mathrm{Nd}_{15} \mathrm{Fe}_{77} \mathrm{~B}_{8}$ magnets with crystalline anisotropy;

- $\mathrm{Fe}-23 \mathrm{Cr}-16 \mathrm{Co}-1 \mathrm{Ti}-1 \mathrm{Nb}$ magnets with shape anisotropy after solution heat treatment, heat treatment in a magnetic field, and low-temperature ageing for $600 \mathrm{~h}$; and

- Alnico magnets with shape anisotropy, containing $4.56 \% \mathrm{Ti}$ and $34 \% \mathrm{Co}$, after standard treatment.

\section{Examination results and their discussion}

$$
\text { 3.1. The } \mathrm{Nd}_{17} \mathrm{Fe}_{75} \mathrm{~B}_{8} \text { alloy }
$$

Examples of magnetic torque curves, obtained for the $\mathrm{Nd}_{17} \mathrm{Fe}_{75} \mathrm{~B}_{8}$ alloy, for several values of the effective magnetic field are shown in Fig. 1. The double curves correspond to the torque moment for two different directions of external magnetic field rotation ( $\mathrm{CW}$ and $\mathrm{ACW})$. The torque curves for $\mathrm{CW}$ and $\mathrm{ACW}$ rotations are identical, when the rotational hysteresis energy is equal to zero.
This is possible in the case, when the external field has too low a value and no losses occur yet (Fig. 1a, the $T(\theta)$ curve is described by the equation $\sin \theta)$, or when the field is sufficiently high and the losses no longer occur (the $T(\theta)$ curve is then described by the equation $\sin 2 \theta$ ). As follows from the curves in Fig. 1d plotted for the field of $H_{e f}=1985 \mathrm{kA} / \mathrm{m}$, a small rotational hysteresis energy still exists.
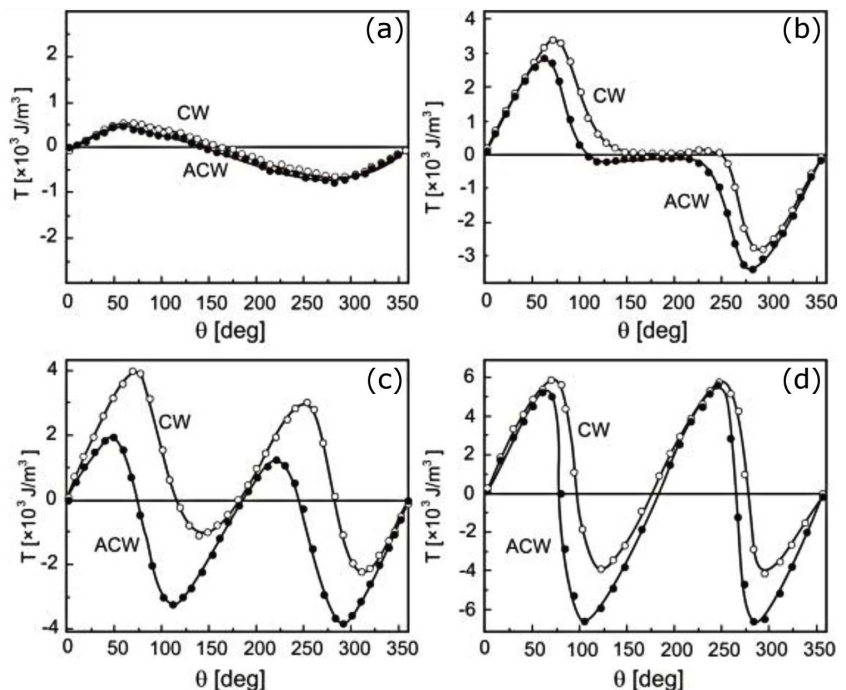

Fig. 1. Torque curves for $\mathrm{Nd}_{17} \mathrm{Fe}_{75} \mathrm{~B}_{8}$ permanent magnet for the following values of effective magnetic field $H_{e f}($ in $\mathrm{kA} / \mathrm{m}$ ): (a) 167, (b) 830, (c) 996, (d) 1985. CW and $\mathrm{ACW}$ denote direction of the external magnetic field rotation, clockwise and anticlockwise, respectively.

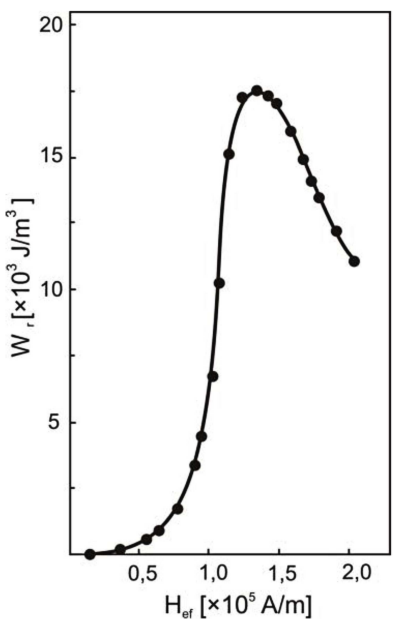

Fig. 2. Rotational hysteresis energy $W_{r}$ vs. effective magnetic field $H_{e f}$ for $\mathrm{Nd}_{17} \mathrm{Fe}_{75} \mathrm{~B}_{8}$ permanent magnet.

Figure 2 represents the dependence of the rotational hysteresis energy, $W_{r}$, on the effective magnetic field, $H_{e f}$. The shape of the $W_{r}\left(H_{e f}\right)$ curve indicate that the rotational hysteresis energy appears already with weak fields. Thus, for $h_{e f}=H_{e f} / H_{A}=0.02, W_{r}=13 \mathrm{~kJ} / \mathrm{m}^{3}$, it attains a maximum value of $\left(W_{r}\right)_{\max }=1710 \mathrm{~kJ} / \mathrm{m}^{3}$ in the field of $h_{e f}=0.16$, and then it slowly decreases. 
The integral of the rotational hysteresis, $R$, for the $\mathrm{Nd}_{17} \mathrm{Fe}_{75} \mathrm{~B}_{8}$ alloy, as calculated based on the obtained rotational hysteresis energy, $W_{r}$, is equal to 0.9 .

Figure 3 shows the variation of coercivity, depending on the angle $\theta$ that is made by the direction of the external magnetic field and the axis of easy magnetization, where the coercivity is expressed by the ratio of the coercivity value for a given angle in the range of $0 \leq \theta \leq 90^{\circ}$ to the coercivity for angle $\theta=0$. The figure displays also theoretical curves for the field of pinning of the domain walls, $H_{P}$, and nucleation, $H_{n}[11]$.

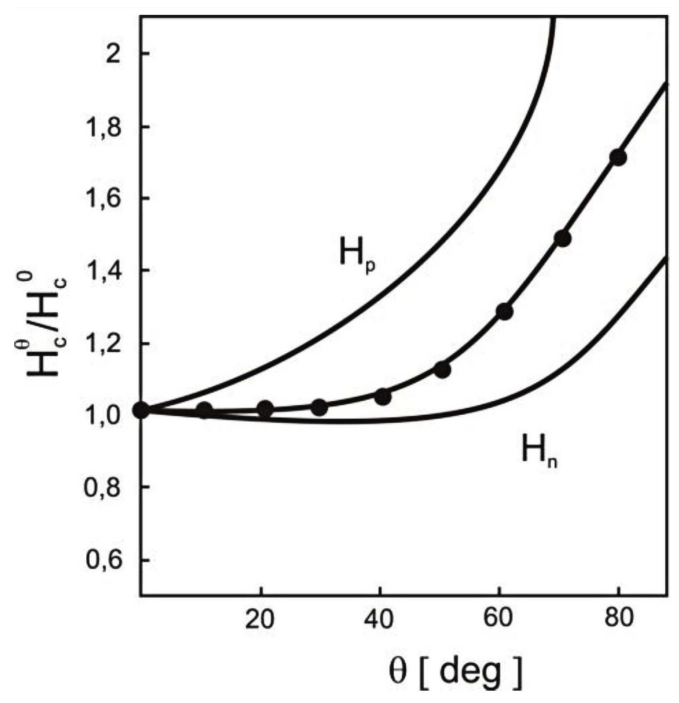

Fig. 3. The angular variation of coercivity $H_{C}^{\theta}$, normalized to its value at $\theta=0$, for $\mathrm{Nd}_{17} \mathrm{Fe}_{75} \mathrm{~B}_{8}$ permanent magnet, compared to the theoretical angular dependence of pinning $H_{P}$ and nucleation $H_{n}$ fields [11].

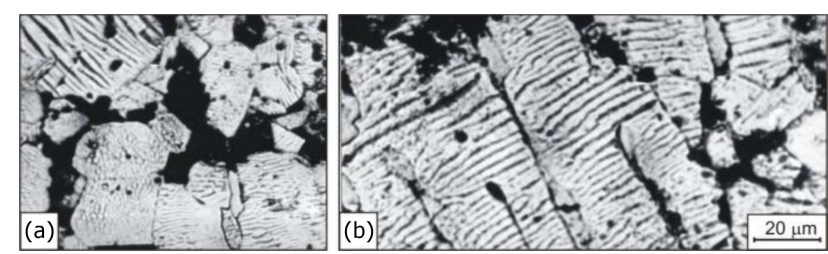

Fig. 4. Domain structure of the $\mathrm{Nd}_{17} \mathrm{Fe}_{75} \mathrm{~B}_{8}$ magnet on basal (a) and axial (b) plane (the powder pattern method).

The domain structure of the $\mathrm{Nd}_{17} \mathrm{Fe}_{75} \mathrm{~B}_{8}$ alloy on the basal (a) and axial (b) plane is represented in Fig. 4. For the majority of grains of the basal plane, the domain structure is composed of a mosaic of stars that represent a picture of wedge-shaped closure domains. In addition to these domains, grains with domain structure composed of $180^{\circ}$ domain walls were observed on the basal plane (Fig. 4a). This indicates that the easy magnetization direction lies in the surface of these grains, thus being perpendicular to the magnetically preferred direction of the whole specimen. On the axial surface, on the other hand, grains with the domain structure composed of $180^{\circ}$ domain walls were mainly observed (Fig. 4b).
The results obtained in this work, which concerns the $\mathrm{Nd}_{17} \mathrm{Fe}_{75} \mathrm{~B}_{8}$ magnet, cannot be fully explained on the grounds of either Stoner-Wohlfarth's theory of singledomain particles (the model of coherent magnetization vector rotation) [12] or Jacobs-Bean's theory (the models of incoherent rotation, referred to as fanning and buckling [13]), or within the more general theory of Shtrikman and Treves for an infinitively long cylinder with a variable radius, which allows for the effect of the incoherent magnetization reversal mechanisms called curling [14].

In the above models, the rotational hysteresis energy appears in a stepwise manner, attaining a maximum value in the field of $h_{e f}=0.5$ in Stoner-Wohlfarth's model and $h_{e f}=0.33$ in Jacobs-Bean's, while in Shtrikman-Treves' model they depend on the reduced radius $S=D / D_{0}$, where $D$ is the cylinder radius and $D_{0}$ is the characteristic radius, and then they decrease to zero at $h_{e f}=1$.

When $S \ll 1$, magnetization process appears by means of coherent magnetization vector rotation, and when $S \gg 1$, it reveals itself through incoherent rotation. In both of the former models, the ratio $\left(W_{r}\right)_{\max } / K$ is equal to 2.67. Whereas, as has been found in the present work for $\mathrm{Nd}_{17} \mathrm{Fe}_{75} \mathrm{~B}_{8}$, the maximum value of rotational hysteresis energy occurs in the field of $h_{e f}=0.16$, while the ratio $\left(W_{r}\right)_{\max } / K_{1}$ is equal to 0.33 , which is not consistent with Stoner-Wohlfarth's and Jacobs-Bean's models. However, the value of the ratio $\left(W_{r}\right)_{\max } / K_{1}$ corresponds to the parameter $S \gg 3$ which, according to ShtrikmanTreves' theory, is indicative of incoherent rotation.

Moreover, the value of the rotational hysteresis integral, $R=0.9$, is higher than the value resulting from Stoner-Wohlfarth's theory $(R=0.42)$, and lower than the integral value from Jacobs-Bean's theory $(R=1.54)$ and is contained in the range from 0.42 to 4.0 calculated by Shtrikman and Treves as a function of the parameter $S$. It follows from the above data that the process of magnetization reversal in the $\mathrm{Nd}_{17} \mathrm{Fe}_{75} \mathrm{~B}_{8}$ alloy consists, most generally, in inhomogeneous rotation. The mechanism of magnetization of the material under consideration is further made more specific by comparing the angular coercivity relationship determined experimentally with the theoretical data (Fig. 3).

This comparison shows that the curve obtained experimentally differs less from the theoretical curve describing magnetization reversal by nucleation [11]. Thus, the process of magnetization in the $\mathrm{Nd}_{17} \mathrm{Fe}_{75} \mathrm{~B}_{8}$ alloy is associated with the mechanism of nucleation rather than pinning.

\subsection{The $\mathrm{Fe}-\mathrm{Cr}-\mathrm{Co}$ alloy}

Figure 5 represents the dependence of rotational hysteresis energy on the effective magnetic field of the Fe$\mathrm{Cr}-\mathrm{Co}$ alloy after complete heat treatment, i.e. heat treatment in the magnetic field and low-temperature ageing for $600 \mathrm{~h}$. The results obtained in the present work can be explained on the grounds of Shtrikman-Treves' theory for an infinitely long cylinder with a variable 


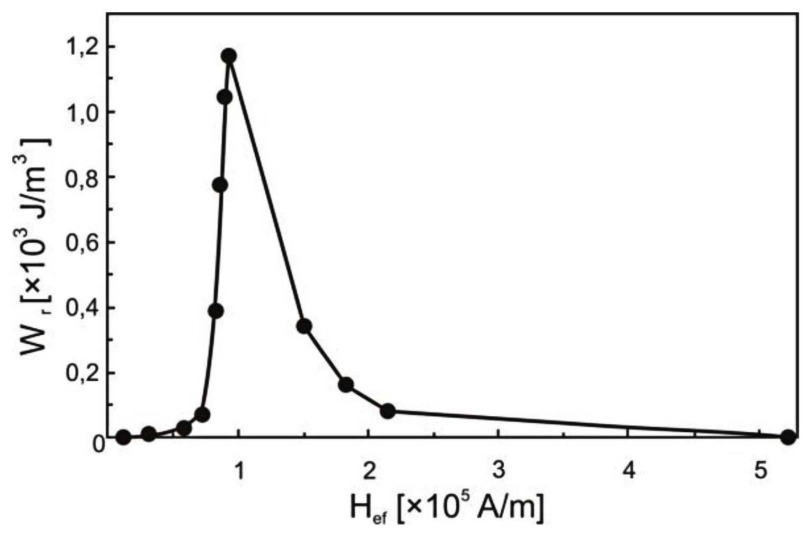

Fig. 5. Rotational hysteresis energy $W_{r}$ vs. effective magnetic field $H_{e f}$ for $\mathrm{Fe}-\mathrm{Cr}-\mathrm{Co}$ permanent magnet.

reduced radius $S$, according to which, when $S>1.47$, the magnetization reversal process occurs by curling [14].

The maximum rotational energy $\left(W_{r}\right)_{\max }$ occurs in the field of $h_{e f}=0.19$ (Fig. 5), which corresponds to the value $S>2$, that is the curling. Also, the $\left(W_{r}\right)_{\max } / K_{1}$ ratio for this alloy has been calculated to be 1.62 . According to Shtrikman-Treves' theory, a reduce radius of $S>2$ corresponds to this value, which provides further evidence for magnetization reversal taking place through the curling. The integral of the rotational hysteresis $R$ for the $\mathrm{Fe}-\mathrm{Cr}-\mathrm{Co}$ alloy, on the other hand, is equal to 1.61, which, according to Shtrikman-Treves' theory, also corresponds to curling [14].

Figure 6 illustrates variations in coercivity as a function of the angle $\theta$ made by the direction of the external magnetic field and the axis of easy magnetization. The shape of the experimental curve is similar to the shape of the theoretical curve for $S \approx 2$, with the maximum value of coercivity occurring at the angle of $\theta \approx 55^{\circ}$ [14]. The obtained value $S \approx 2$ also confirms the fact that the magnetization reversal process occurs in the $\mathrm{Fe}-\mathrm{Cr}-\mathrm{Co}$ alloy through the curling.

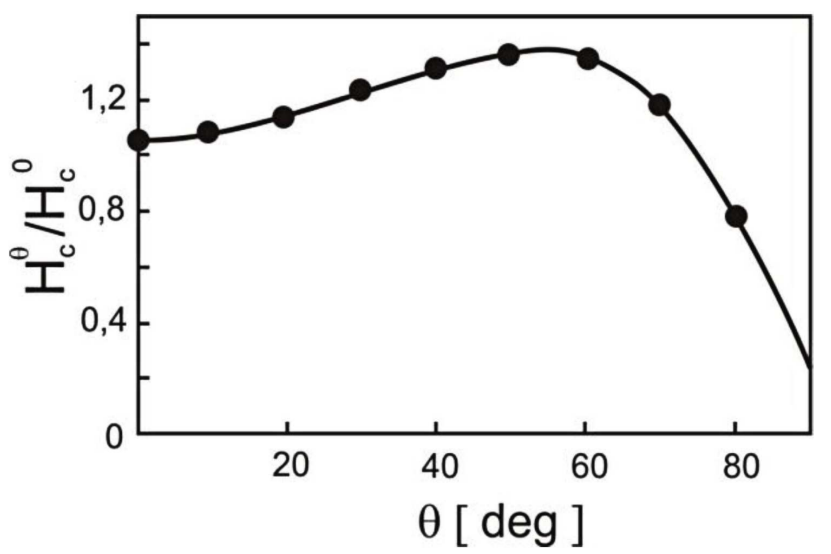

Fig. 6. The variation of coercivity $H_{C}^{\theta}$ normalized to its value at $H_{C}^{0}$ as a function of the angle $\theta$ made by the direction of the external magnetic field with the axis of easy magnetization in $\mathrm{Fe}-\mathrm{Cr}-\mathrm{Co}$ alloy.
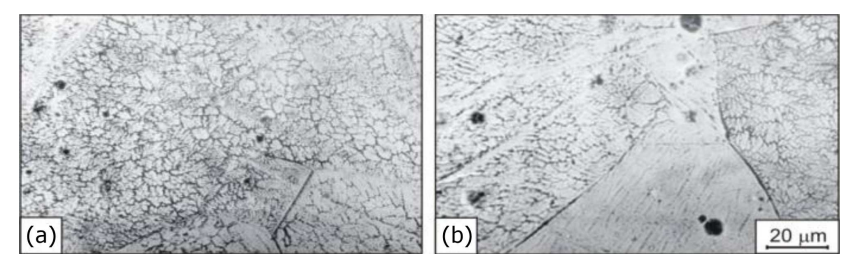

Fig. 7. The domain structures for two mutually perpendicular surfaces: parallel (a) and perpendicular (b) to the casting direction of the $\mathrm{Fe}-23 \mathrm{Cr}-16 \mathrm{Co}-1 \mathrm{Ti}-$ $1 \mathrm{Nb}$ alloy after homogenization (the powder pattern method).

Figure 7 shows typical domain structures for two mutually perpendicular surfaces: parallel to the direction of casting (the axial plane, Fig. 7a), and perpendicular (the basal plane, Fig. 7b), after homogenization.

After this heat treatment process, the alloy constitutes a supersaturated solid solution with a body-centred cubic lattice. In spite of this, the domain structure of this alloy, as shown in Fig. 7, is typical for uniaxial ferromagnetic materials (e.g. Co) and is composed of "stars", "flowers", "labyrinths", or domains with $180^{\circ}$ Bloch walls. This domain structure picture results, among other things, from the mutual relationship between the anisotropy and magnetostriction constants.

This domain structure undergoes a significant change upon heat treatment in the magnetic field (Fig. 8). This change is that a domain structure with typical Bloch walls transforms into the so-called interaction domain structure [15]. After the heat treatment, flat-parallel domains are observed (Fig. 8a) on the axial plane, in which the magnetization vector is close to the direction of the external magnetic field applied during heat treatment. On the basal plane, that is the one perpendicular to the magnetically preferred direction, "labyrinth"shaped domains are observed (Fig. 8b). It is interesting to note that the "labyrinth" domains form concentric circles in each of the grains on the basal plane (Fig. 8b). On the axial plane, domains with $180^{\circ}$ walls are observed (Fig. 8a), which have the identical crystallographic orientation within a single grain, often passing continuously through the grain boundaries, and their width is not dependent on the grain size. Continued low-temperature ageing results in a refinement of the structure of the concentric circle-shaped domains observed on the basal plane and a reduction of the width of the $180^{\circ}$ domains on the axial plane.

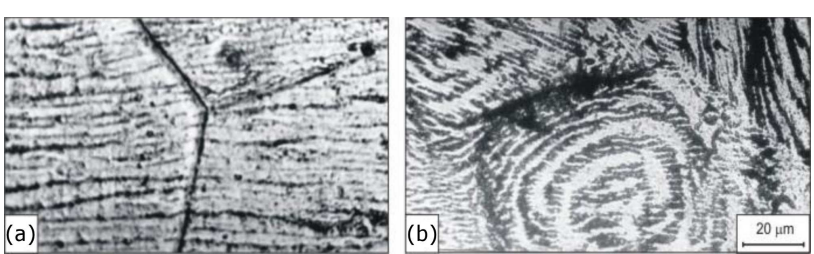

Fig. 8. Comparison of the domain structure of the $\mathrm{Fe}-$ $23 \mathrm{Cr}-16 \mathrm{Co}-1 \mathrm{Ti}-1 \mathrm{Nb}$ alloy after heat treatment in the magnetic field taken from the plane paralel (a) and perpendicular (b) to the magnetically preferred direction (the powder pattern method). 


\subsection{The Alnico alloy}

Figure 9 represents the dependence of the rotational hysteresis energy $W_{r}$ on the effective magnetic field $H_{e f}$ for the Alnico alloy.

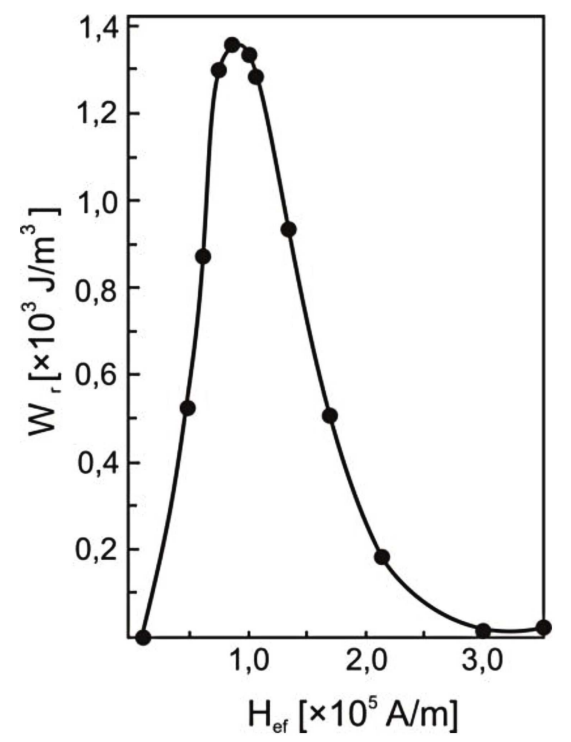

Fig. 9. Rotational hysteresis energy $W_{r}$ vs. effective magnetic field $H_{e f}$ for Alnico alloy.

The behaviour of the curve indicates that the rotational hysteresis energy occurs already in the field of $H_{e f}=24 \mathrm{kA} / \mathrm{m}\left(h_{e f}=0.125\right)$ and attains a maximum value of $\left(W_{r}\right)_{\max }=14.2 \times 10^{4} \mathrm{~J} / \mathrm{m}^{3}$ in the field of $H_{e f}=80 \mathrm{kA} / \mathrm{m}\left(h_{e f}=0.37\right)$, and then decreases to zero in the field of $H_{e f}=288 \mathrm{kA} / \mathrm{m}$. Due to the fact that the single-domain particles in the Alnico alloy have an oblong shape with a relatively small diameter, Shtrikman-Treves' theory [14] was used to explain the process of magnetization reversal of this alloy. According to this theory, the magnetic field value, in which the maximum energy occurs $\left(h_{e f}=0.37\right)$, corresponds to the reduced radius $S=1.6$, which suggests that the magnetization reversal process occurs through the curling.

This is also confirmed by the value of the rotational hysteresis integral calculated for this alloy, which is $R=1.4$. The value corresponding to the value of this integral $(R=1.4)$ is $S=1.7$. Moreover, according to Shtrikman-Treves' theory, the $S$ parameter value corresponding to the value of this integral $(R=1.4)$ is $S=1.7[14]$.

Figure 10 shows variations of coercivity as a function of the angle $\theta$ made by the direction of the external magnetic field and the axis of easy magnetization. The shape of the experimental curve is similar to the shape of the theoretical curve for $S \approx 1.7$, with the maximum coercivity value occurring at the angle of $\theta \approx 55^{\circ}$ [14]. The obtained value $S \approx 1.7$ also confirms that the magnetization reversal process occurs through the curling.

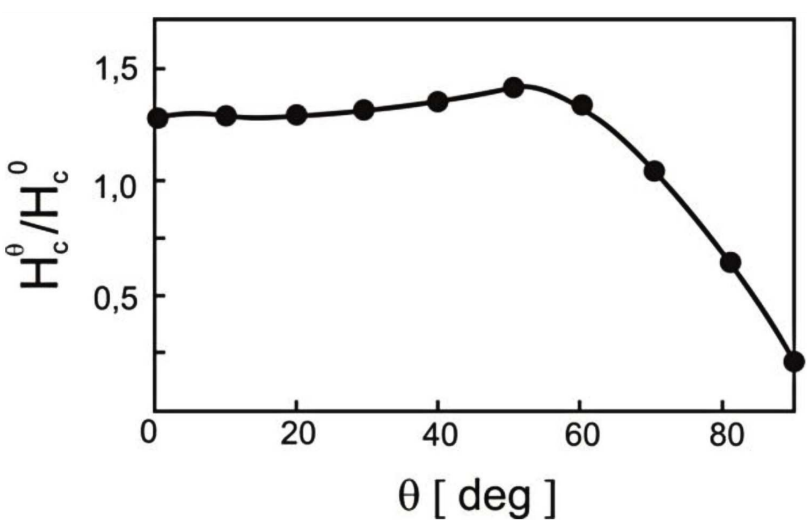

Fig. 10. The variation of coercivity $H_{C}^{\theta}$ normalized to its value at $H_{C}^{0}$ as a function of the angle $\theta$ made by the direction of the external magnetic field with the axis of easy magnetization in Alnico alloy.

The reduced radii $S$ determined for the maximum energy losses $(S=1.6)$, the integral of rotational hysteresis $(S=1.7)$ and angular coercivity relationship $(S \approx 1.7)$ have similar values, which provides additional evidence for the correctness of the selection of curling as the Alnico alloy magnetization reversal mechanism.
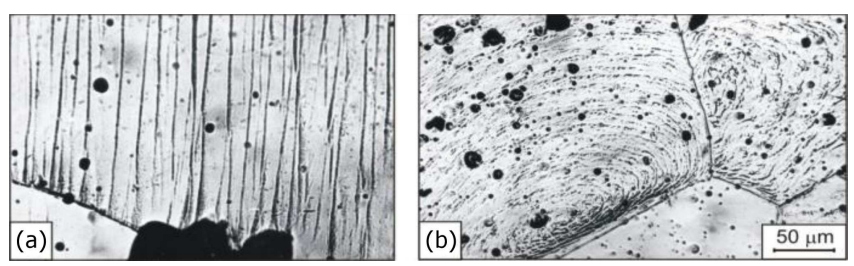

Fig. 11. The domain structure of the Alnico alloy $(4.56 \% \mathrm{Ti}, 34 \% \mathrm{Co})$ after standard heat treatment observed on the sample surface parallel (a) and perpendicular (b) to the easy axis of magnetization (the powder pattern method).

Figure 11 compares the domain structures from the surface, parallel (a) and (b) perpendicular to the magnetically preferred direction, respectively. The domain structure observed on the axial plane is composed of $180^{\circ}$ domain walls passing continuously through the grain boundary (Fig. 11a). The domain structure on the basal plane (Fig. 11b), on the other hand, is composed of concentric circles observed in each of the grains.

\section{Concluding remarks and findings}

Basing on the performed examination of the torque curves, rotational hysteresis energy and its integral, angular coercivity relationship and the domain structure of magnetically hard materials characterized by different types of magnetic anisotropy, and comparison of the obtained results with different models of the magnetization reversal process, it has been found that: 
- in the $\mathrm{Nd}_{17} \mathrm{Fe}_{75} \mathrm{~B}_{8}$ alloy, being an example of material with crystalline anisotropy, the magnetization reversal process consists in the nucleation and growth of domains with the opposite direction of the magnetization vector. The domain structure image is typical for materials of a single magnetically preferred direction;

- in the $\mathrm{Fe}-23 \mathrm{Cr}-16 \mathrm{Co}-1 \mathrm{Ti}-1 \mathrm{Nb}$ alloy, being an example of material with shape anisotropy, the magnetization reversal process consists in the incoherent rotation of the magnetization vector, which, by Shtrikman-Treves' theory, is referred to as curling. Moreover, the existence of so-called interaction domains in this alloy, after being heat treated in the magnetic field, has been confirmed experimentally;

- in the Alnico alloy, also being an example of material with shape anisotropy, the magnetization reversal process consists also in the incoherent rotation of the magnetization vector, which, by ShtrikmanTreves' theory, is referred to as curling.

\section{References}

[1] M. Leonowicz, J.J. Wysłocki, Modern Hard Magnetic Materials. Technology, Coercivity Mechanism, Application, Wydawnictwa Naukowo-Techniczne, Warsaw 2005 (in Polish).

[2] R.A. McCurrie, in: Ferromagnetic Materials, Ed. E.P. Wohlfarth, Vol. 3, North-Holland, 1982, p. 107.
[3] Y.L. Sun, J.T. Zhao, Z. Liu, W.X. Xia, A.R. Yan, J. Magn. Magn. Mater. 379, 58 (2015).

[4] H. Kaneko, M. Homma, N. Nakamura, G. Thomas, IEEE Trans. Magn. MAG-13, 1325 (1977).

[5] D.J. Craik, E.W. Hill, J. Phys. (Paris) 38, C1-39 (1977).

[6] M. Sagawa, S. Fujimura, M. Togawa, H. Yamamoto, Y. Matsuura, J. Appl. Phys. 55, 2083 (1984).

[7] M. Katter, Ph.D. Thesis, Technische Universität, Wien 1991.

[8] H. Kronmüller, K.-D. Durst, M. Sagawa, J. Magn. Magn. Mater. 74, 291 (1988).

[9] R.M. Bozorth, Ferromagnetism, IEEE Press (Classic Reissue), New York 1993.

[10] I.S. Jacobs, F.E. Luborsky, J. Appl. Phys. 28, 467 (1957).

[11] H. Kronmüller, K.D. Durst, G. Martinek, J. Magn. Magn. Mater. 69, 149 (1987)

[12] E.C. Stoner, E.P. Wohlfarth, Philos. Trans. R. Soc. Lond. 240A, 599 (1948).

[13] I.S. Jacobs, C.P. Bean, Phys. Rev. 100, 1060 (1955).

[14] S. Shtrikman, T. Treves, J. Phys. Rad. 20, 286 (1959).

[15] D.J. Craik, Z. Angew. J. Phys. (Paris) 21, 1 (1966). 